\title{
Factors Influencing Elevated Mortality Rates of Patients with Schizophrenia Hospitalized with COVID
}

\author{
Kathleen A. Crapanzano ${ }^{1}$ (1) - Sydney Smith ${ }^{1} \cdot$ Rebecca Hammarlund $^{1,2}$
}

Received: 12 August 2021 / Accepted: 11 November 2021 / Published online: 20 November 2021

(c) The Author(s), under exclusive licence to Springer Science+Business Media, LLC, part of Springer Nature 2021

\begin{abstract}
Work completed since the pandemic began has repeatedly demonstrated elevated mortality rates in people with schizophrenia hospitalized with COVID. They are a vulnerable group due to multiple issues-for example high co-morbidity rates of medical illness, often impaired insight and judgment, barriers to obtaining health care, and trouble understanding and implementing preventive measures. The objective of this study was to evaluate if a diagnosis of schizophrenia in the context of COVID-19 requiring hospitalization increased the risk for people with a diagnosis of schizophrenia to be intubated, admitted to the ICU or die when compared to people hospitalized with COVID-19 who did not have schizophrenia. This was accomplished by doing a retrospective chart review of 123 people with schizophrenia and matched controls. Although we found elevated rates of these outcomes in the patients with schizophrenia, our analysis attributed these differences to congregate living, rather than the illness itself.
\end{abstract}

Keywords COVID $\cdot$ Schizophrenia $\cdot$ Mortality rates $\cdot$ Congregate settings

\section{Introduction}

First declared a pandemic in March 2020 (The New York Times, 2020), the novel SARS-CoV-2/Coronavirus-19 (COVID-19) infection has since claimed over 609,000 lives in the United States alone (Center for Disease Control \& Prevention, 2021b). From the beginning, public health officials were concerned with identifying those populations that might be most at risk for severe infection and death. The Center for Disease Control's June 2020 list of groups at risk of severe COVID-19 infections included adults over 65 years of age and people with long standing systemic health and social inequalities, including people with disabilities (Center for Disease Control \& Prevention, 2021a). People with schizophrenia fall into this category for several reasons including the fact that they are more likely to

Kathleen A. Crapanzano

Kcrap1@1suhsc.edu

1 Department of Psychiatry, Louisiana State University Health Sciences Center, Baton Rouge, Medical Education and Innovation Building, 5246 Brittany Drive, RM 340, Baton Rouge, LA 70808, USA

2 Our Lady of the Lake Office of Research, 7777 Hennessy Blvd, Suite 6004, Baton Rouge, LA 70808, USA have multiple poorly treated co-morbidities (Laursen et al., 2012), difficulty communicating symptoms of illness (Pary \& Barton, 1988), poor insight and judgement (Kozloff et al., 2020), barriers to obtaining healthcare (Kozloff et al., 2020), and trouble understanding and implementing preventative measures (Kozloff et al., 2020).

In the case of COVID-19 infection, people with schizophrenia may be at particularly high risk for severe infection for specific socioeconomic and medical reasons. First, people with schizophrenia are over-represented in vulnerable populations living in congregate settings, including prisoners, nursing home residents, and mental health facility residents (Kozloff et al., 2020). Congregate living is a risk factor for severe infection because these settings rarely lend themselves easily to enacting recommended social distancing protocols, making exposure to the illness more likely (Kozloff et al., 2020).

Living situations aside, people with schizophrenia are also more likely to have severe COVID-19 infections due to the presence of comorbidities, such as cardiovascular disease, and chronic obstructive pulmonary disorder that are common in people with schizophrenia (Brink et al., 2019) and have been linked with exacerbated COVID-19 symptoms (Jordan et al., 2020). Patients with schizophrenia are not only more likely to have one of these conditions, they 
are more likely to have multiple comorbidities (Brink et al., 2019) and for these comorbidities to be poorly controlled at baseline (Brink et al., 2019). Thus, even before COVID19 infection is considered, people with schizophrenia are at increased risk of premature death from comorbid diabetes mellitus, cardiovascular and respiratory diseases (Brink et al., 2019; Hoang et al., 2011; Olfson et al., 2015).

Previous work has confirmed the suspicion that people with schizophrenia may be at higher risk of severe COVID19 infection, finding an increased risk of mortality in individuals with the virus and a diagnosis of schizophrenia (Fond et al., 2021b; Nemani et al., 2021). Other work has suggested that patients with serious mental illness, including schizophrenia, may have a more difficult hospital course in addition to the increased risk of mortality. In a recent Korean study, patients with severe pre-existing mental illness were more likely to have severe COVID-19 outcomes (death, admission to an intensive care unit or intubation) than patients without a history of severe mental illness (Lee et al., 2020).

Research since the start of the pandemic has explored the clinical course of patients with schizophrenia who contracted COVID-19 and required higher levels of medical care, specifically inpatient hospitalization and ICU admission. The purpose of this project was to investigate the associations between patients with schizophrenia and the COVID-19 clinical outcomes of death, intubation, or ICU admission. The objective of the present study was to evaluate if a diagnosis of schizophrenia increased the risk for a hospitalized patient with COVID-19 to be intubated, admitted to the ICU or die, compared to people with COVID-19 who did not have schizophrenia.

\section{Methods}

This retrospective chart review was approved by the Institutional Review Board of Louisiana State University Health Sciences Center New Orleans.

\section{Sample and Procedure}

A list of potential patients was extracted from a larger registry of patients of a large health system in the Southern United States who tested positive for COVID-19 throughout the 2020 calendar year. The lead research scientist in charge of the registry pulled any patient in the registry that had schizophrenia or schizoaffective disorder listed in their medical history. The list of potential patients was entered into REDCap and data was gathered on all patients on the list.

Once the data were collected, patients were assessed for inclusion in the final data set. Patients were included if (1) a diagnosis of schizophrenia or schizoaffective disorder could be found in their electronic medical record and (2) they were admitted to a hospital within the health system while they were COVID-19 positive, even if the primary Emergency Room or hospital diagnosis was something other than COVID-19.

After all patients in the MI group (i.e., patients with schizophrenia or schizoaffective disorder) were identified, the registry was hand searched for matched controls (MC) for each MI patient on the basis of age, sex, race, ethnicity, and BMI. MC patients also had to have been admitted while COVID-19 positive. In a minority of cases, admitted MC patients of the exact same age could not be found. For such cases, an otherwise appropriate match was found within \pm 2 years of age. Regarding BMI, an exact match was largely not possible. Instead, MC patients were selected if their BMI was within \pm 5 of the MI patient's BMI.

Once all MC patients were identified, the remaining data for these patients was gathered. Finally, data entry was rechecked for all MI patients.

\section{Data Analyses}

Frequencies, means, and standard deviations were calculated to describe the data. Independent samples t-tests were used to ensure the two patient groups did not differ on age or BMI, two match variables that had some minor variability. Chi-square analyses were used to determine if patient group (MI vs. MC) was related to any of the following three yes/no outcomes: admission to the ICU, intubation, or a deceased discharge disposition. Chi-square analyses were also used to determine if smoking status and congregate living were related to the three outcomes. Finally, a binary logistic regression was conducted to assess the relationship between patient group and deceased discharge disposition while accounting for the relationship between congregate living and the same outcome.

\section{Results}

\section{Sample and Demographics}

The original registry list contained 201 patients. Upon review, 16 of these did not have a diagnosis of schizophrenia or schizoaffective disorder in their chart. Of the remaining 185 patients, 62 were not admitted to the hospital while they were diagnosed with COVID-19. Thus, 123 patients (79 female; 64\%) were included in the database. A further 123 matched-control patients were identified from the registry. Thus the $\mathrm{N}$ for the entire sample was 246 (158 female). Mean age was $60(\mathrm{SD}=14.13)$, with a range from 22 to 85 years. Age did not differ between groups, $t(244)=-0.03$, $p=0.975$. Average BMI was $30.63(\mathrm{SD}=8.83)$ and did not 
differ between the MI and MC patient groups, $t(243)=0.34$, $p=0.735$. Sixty-four percent of the sample were Black/ African American, 32\% were white, 2\% were Asian, and the remaining $2 \%$ were other, multiple, or unknown races. Regarding ethnicity, 95\% were non-Hispanic, $2 \%$ were Hispanic, and $2 \%$ were unknown.

\section{Mental Health Status and Outcomes}

The ICU admission rate was $46 \%$ for the MI group and $38 \%$ for the MC group. Patient group was not significantly related to being admitted to the ICU during one's hospital stay, $\chi^{2}(1)=1.35, p=0.245$.

The intubation rate was $24 \%$ for the MI group and $17 \%$ for the MC group. Patient group was not significantly related to being intubated during one's hospital stay, $\chi^{2}(1)=1.61$, $p=0.205$.

The rate of deceased discharge status was $24 \%$ in the MI group and $14 \%$ in the MC group. Patient group was marginally related to being deceased at discharge, $\chi^{2}(1)=3.85$, $p=.050$. Five and four percent of the MI and MC groups were discharged to hospice care, and $7 \%$ of each group died at some point after discharge but before data collection was completed.

\section{Comorbidities and Outcomes}

Table 1 displays frequencies and test statistics of eight specific comorbidities within the MI and MC groups. Only chronic obstructive pulmonary disease (COPD) differed by group, with a higher frequency in the MI group $(n=20$ vs. $\mathrm{n}=9$ in $\mathrm{MC}$ group).

Table 1 also displays the test statistics of the eight specific comorbidities in relation to ICU admission, intubation, and death at discharge. Only chronic kidney disease (CKD) was related to ICU admission; $56.1 \%(n=23$ of 41$)$ of patients with CKD were admitted to the ICU, compared with $39.0 \%$ ( $n=80$ of 205) of those without CKD.

None of the eight comorbidities were significantly related to being intubated during the encounter.

Death at discharge was marginally related to hypertension and significantly related to diabetes. For hypertension, $21.8 \%$ ( $\mathrm{n}=38$ of 174$)$ were deceased upon discharge compared with $11.1 \%$ ( $\mathrm{n}=8$ of 72 ) in those without hypertension. For diabetes, $25.5 \%(n=25$ of 98$)$ were deceased upon discharge, compared with $14.2 \%(n=21$ of 148$)$ in those without diabetes.

\section{Smoking Status and Outcomes}

Twelve patient records had uncertain smoking status information, thus these cases were excluded from analyses. Fifty percent $(n=56$ of 113) of the MI group reported past or current smoking, in comparison to $37 \%(\mathrm{n}=45$ of 121$)$ in the MC group. Patient group was not significantly related to smoking status, $\chi^{2}(1)=3.64, p=0.056$.

The ICU admission rate was $38 \%$ for non-smokers and $44 \%$ for current or past smokers. Smoking status was not significantly related to ICU admission, $\chi^{2}(1)=0.85, p=0.356$.

The intubation rate was $19 \%$ for non-smokers and $21 \%$ for current or past smokers. Smoking status was not significantly related to intubation, $\chi^{2}(1)=0.15, p=0.704$.

The rate of deceased discharge status was $15 \%$ for nonsmokers and $22 \%$ for current or past smokers. Smoking status was not significantly related to being deceased at discharge, $\chi^{2}(1)=1.77, p=0.183$.

\section{Congregate Living and Outcomes}

Sixteen patient records had uncertain congregate living status information, thus these cases were excluded from analyses. Eighty-three percent $(n=96$ of 115) of the MI group
Table 1 Comorbidity frequencies, Chi-square and p-values by patient group

\begin{tabular}{|c|c|c|c|c|c|c|c|c|c|c|c|c|}
\hline & \multicolumn{4}{|c|}{$\begin{array}{l}\text { Comorbidity frequen- } \\
\text { cies }\end{array}$} & \multicolumn{8}{|c|}{ Chi-square $\left(\chi^{2}\right)$ test statistics and probability values (p-value) } \\
\hline & \multicolumn{2}{|c|}{ MI group } & \multicolumn{2}{|c|}{ MC group } & \multicolumn{2}{|c|}{ Group } & \multicolumn{2}{|c|}{$\begin{array}{l}\text { ICU admis- } \\
\text { sion }\end{array}$} & \multicolumn{2}{|c|}{ Intubation } & \multicolumn{2}{|c|}{ Death } \\
\hline & Yes & No & Yes & No & $\chi^{2}$ & $\mathrm{p}$-value & $\chi^{2}$ & p-value & $\chi^{2}$ & p-value & $\chi^{2}$ & p-value \\
\hline Cancer & 4 & 119 & 9 & 114 & 2.03 & 0.154 & 0.10 & 0.748 & 0.21 & 0.649 & 0.17 & 0.677 \\
\hline Hypertension & 91 & 32 & 83 & 40 & 1.26 & 0.262 & 2.14 & 0.144 & 1.60 & 0.206 & 3.86 & 0.050 \\
\hline Heart failure & 16 & 107 & 10 & 113 & 1.55 & 0.213 & 0.14 & 0.710 & 1.96 & 0.162 & 2.79 & 0.095 \\
\hline Kidney disease & 20 & 103 & 21 & 102 & 0.03 & 0.864 & 4.09 & 0.043 & 2.43 & 0.119 & 3.62 & 0.057 \\
\hline Asthma & 8 & 115 & 9 & 114 & 0.06 & 0.802 & 1.17 & 0.281 & 0.08 & 0.776 & 1.97 & 0.160 \\
\hline COPD & 20 & 103 & 9 & 114 & 4.73 & 0.030 & 0.12 & 0.731 & 0.87 & 0.352 & 0.64 & 0.424 \\
\hline Liver Disease & 1 & 122 & 1 & 122 & 0.00 & 1.000 & 0.05 & 0.816 & 0.91 & 0.339 & 1.00 & 0.316 \\
\hline Diabetes & 56 & 67 & 42 & 81 & 3.32 & 0.068 & 0.61 & 0.433 & 2.70 & 0.100 & 4.97 & 0.026 \\
\hline
\end{tabular}

Significant p-value is indicated in bold 
and $18 \%(n=21$ of 115$)$ of the MC group were admitted from congregate living situations. Patient group was significantly related to congregate living situation, $\chi^{2}(1)=97.86$, $p<.001$.

The ICU admission rate was $38 \%$ for persons not living in congregate settings and $45 \%$ for persons living in congregate settings. Congregate living status was not significantly related to ICU admission, $\chi^{2}(1)=1.24, p=0.265$.

The intubation rate was $18 \%$ for persons not living in congregate settings and $23 \%$ for persons living in congregate settings. Congregate living status was not significantly related to intubation status, $\chi^{2}(1)=1.02, p=0.312$.

The rate of deceased discharge status was $11 \%$ for persons not living in congregate settings and $27 \%$ for persons living in congregate settings. Congregate living status was significantly related to being deceased at discharge, $\chi^{2}(1)=9.53$, $p=.002$.

\section{Other Demographic Variables and Outcomes}

Sex was not related to ICU admission, $\chi^{2}(1)=.10, p=.756$, intubation, $\chi^{2}(1)=.14, p=.713$, or death at discharge, $\chi^{2}(1)=.28, p=.598$.

Race (Black vs. white) was not related to ICU admission, $\chi^{2}(1)=.09, p=.769$, intubation, $\chi^{2}(1)=3.14, p=.076$, or death at discharge, $\chi^{2}(1)=.10, p=.758$.

\section{Congregate Living, Mental Health Status, and Death at Discharge}

Table 2 contains results of a binary logistic regression with patient group, congregate living status, hypertension, and diabetes predicting deceased status at discharge.

\section{Discussion}

Previous work has reported that people with schizophrenia died at a higher rate than people without schizophrenia when hospitalized for COVID-19 (Fond et al., 2021a; Lee et al., 2020; Nemani et al., 2021). This study set out to determine what factors might influence this disparate outcome. While our study also found a higher death rate from COVID-19 when a person was diagnosed with schizophrenia, the results were not statistically significant, likely due to our relatively small sample size. The ICU admission rate and intubation rates were likewise higher in people with schizophrenia, although again not statistically significant. However, these two results make differential decision making or withheld care a less likely explanation for the increased mortality rate.

Obesity, gender, race and smoking were controlled in the paired data. Of note, smoking status may not have been an accurate measure given that with the acuity of the cases, documenting smoking status may not have been the highest priority. Of the comorbid conditions, COPD was overrepresented in the group of patients with schizophrenia, but the others were distributed evenly in the matched groups.

The one issue that did predict mortality was pre-admission congregate living-independently and when the diagnosis of schizophrenia was controlled. Possible explanations for the higher mortality rate specifically in congregate settings could include higher exposure to the virus when living in close quarters, limited ability for the congregate setting to isolate people who were positive for COVID, potential delay in treatment due to staff not being aware of the severity of symptoms or perhaps patient unawareness of the need for intervention. In one study, staff were also suspected to be the vector for introducing COVID-19 into the facility (Terebuh et al., 2021). Of note, in spite of the knowledge that people with schizophrenia were dying at higher rates than the general population and that the virus spread more quickly in congregate living situations, people in these two populations were not prioritized for receiving the vaccine.

Congregate living (whether in a group home, nursing home, jail/prison, or state psychiatric facility) is currently not in favor with mental health advocates. Community services and supported housing allow for more freedom and better quality of life for people with schizophrenia, although these resources are often not universally available. Our study offers yet another reason for communities to consider alternatives to congregate living.

The primary limitation to our study was our sample size. Although we found differences in our outcomes, the numbers weren't big enough to reach statistical significance. Given the tedious nature of reviewing the charts by hand to extract the data, it will be difficult to get a data base large
Table 2 Binary logistic regression analysis

\begin{tabular}{lrlllllll}
\hline Predictor & \multicolumn{1}{c}{$S$} & $S E \beta$ & Wald's $\chi^{2}$ & $d f$ & $p$ & $e \beta$ & \multicolumn{2}{c}{$95 \%$ CI for $\mathrm{e} \beta$} \\
\hline Constant & -2.631 & 0.449 & 34.299 & 1 & $<.001$ & 0.072 & - & - \\
Hypertension & 0.528 & 0.443 & 1.418 & 1 & 0.234 & 1.695 & 0.711 & 4.038 \\
Diabetes & 0.526 & 0.353 & 2.228 & 1 & 0.136 & 1.693 & 0.848 & 3.380 \\
Congregate setting & 0.690 & 0.335 & 4.240 & 1 & $\mathbf{0 . 0 3 9}$ & 1.993 & 1.034 & 3.842 \\
Patient group & 0.142 & 0.395 & 0.129 & 1 & 0.719 & 1.152 & 0.532 & 2.499 \\
\hline
\end{tabular}

221.796 (-2 Log Likelihood), 0.055 (Cox \& Snell), 0.088 (Nagelkerke, Model $\chi^{2}(1)=2.995, p=0.885$ ) Significant p-value is indicated in bold 
enough to determine if the observed differences are meaningful statistically. This study is also limited in that it only evaluates outcomes among individuals admitted to the hospital. For this reason, study results should not be used to draw conclusions about the overall outcomes of COVID-19 in patients with schizophrenia or schizoaffective diagnoses. Future research should continue to look at differences in health outcomes for people with chronic mental illness and the reasons for those differences.

In summary, our study found higher death rates from people hospitalized with COVD and diagnosed with schizophrenia as have other studies, but determined the primary cause was congregate living status as opposed to being related to the diagnosis of schizophrenia.

Author Contributions All authors contributed to the study conception and design. Material preparation, data collection and analysis were performed by Rebecca Hammarlund and Sydney Smith. The first draft of the manuscript was written by Sydney Smith, Rebecca Hammarlund and Kathleen Crapanzano, and all authors commented on previous versions of the manuscript. All authors read and approved the final manuscript.

Funding No outside sources of funding was received for conducting this study or to assiet in the preparation of the manuscript.

\section{Declarations}

Conflict of interest The authors have no relevant financial or non-financial interests to disclose.

Ethical Approval The study was approved by the Institutional Review Board of Louisiana State University Health Sciences Center in New Orleans.

\section{References}

Brink, M., Green, A., Bojesen, A., et al. (2019). Excess medical comorbidity and mortality across the lifespan in schizophrenia: A nationwide Danish register study. Schizophrenia Research, 206, 347-354.

Center for Disease Control and Prevention. (2021a). People with certain medical conditions. Retrieved July 28, 2021, from https:// www.cdc.gov/coronavirus/2019-ncov/need-extra-precautions/ people-with-medical-conditions.html

Center for Disease Control and Prevention. (2021b). United States COVID-19 cases, deaths, and laboratory testing (NAATs) by state, territory, and jurisdiction. Retrieved July 28, 2021, from https:// covid.cdc.gov/covid-data-tracker/\#cases_casesper100klast7days/

Fond, G., Pauly, V., Leone, M., et al. (2021a). Disparities in intensive care unit admission and mortality among patients with schizophrenia and COVID-19: A national cohort study. Schizophrenia Bulletin, 47(3), 624-634.

Fond, G., Pauly, V., Orleans, V., et al. (2021b). Increased in-hospital mortality from COVID-19 in patients with schizophrenia. Encephale, 47(2), 89-95.

Hoang, U., Stewart, R., \& Goldacre, M. J. (2011). Mortality after hospital discharge for people with schizophrenia or bipolar disorder: Retrospective study of linked English hospital episode statistics, 1999-2006. BMJ, 343, d5422.

Jordan, R. E., Adab, P., \& Cheng, K. K. (2020). Covid-19: Risk factors for severe disease and death. BMJ, 368, m1198.

Kozloff, N., Mulsant, B. H., Stergiopoulos, V., \& Voineskos, A. N. (2020). The COVID-19 global pandemic: Implications for people with schizophrenia and related disorders. Schizophrenia Bulletin, 46(4), 752-757.

Laursen, T. M., Munk-Olsen, T., \& Vestergaard, M. (2012). Life expectancy and cardiovascular mortality in persons with schizophrenia. Current Opinion in Psychiatry, 25, 83-88.

Lee, S. W., Yang, J. M., Moon, S. Y., et al. (2020). Association between mental illness and COVID-19 susceptibility and clinical outcomes in South Korea: A nationwide cohort study. The Lancet, 7, 1025-1031.

Nemani, K., Li, C., Olfson, M., et al. (2021). Association of psychiatric disorders with mortality among patients with COVID-19. JAMA Psychiatry, 78(4), 380-386.

Olfson, M., Gerhard, T., Huang, C., et al. (2015). Premature mortality among adults with schizophrenia in the United States. JAMA Psychiatry, 72(12), 1172-1181.

Pary, R. J., \& Barton, S. N. (1988). Communication difficulty of patients with schizophrenia and physical illness. Southern Medical Journal, 81(4), 489-490.

Terebuh, P. D., Egwiekhor, A. J., Gullett, H. L., et al. (2021). Characterization of community-wide transmission of SARS-CoV-2 in congregated living settings and local public health-coordinated response during the initial phase of the COVID-19 pandemic. Influenza and Other Respiratory Viruses, 15, 439-445.

The New York Times. (2020). Coronavirus live updates: W.H.O. declares pandemic as number of infected countries grows. The New York Times. Retrieved July 28, 2020, from https://www.nytim es.com/2020/03/11/health/coronavirus-pandemic-who.html

Publisher's Note Springer Nature remains neutral with regard to jurisdictional claims in published maps and institutional affiliations. 